\title{
Zwischen Placebo und Erfolgsmodell. Direkte Demokratie auf der Landesebene
}

\author{
Frank Decker*
}

Während direktdemokratische Instrumente auf Bundesebene weiterhin fehlen, gehören sie heute zum institutionellen Kernbestand sämtlicher deutschen Landes- und Kommunalverfassungen. Die plebiszitäre Enthaltsamkeit des Grundgesetzes mag mit Blick auf die Kommunen gut erklärbar sein; mit Blick auf die Länder ist sie aber erstaunlich, da sich die institutionellen Strukturen der Regierungssysteme auf beiden Ebenen ansonsten stark ähneln. Die abweichende Entwicklung war in der Geschichte der Bonner Republik von Beginn an angelegt. Nicht nur, dass alle 13 vorgrundgesetzlichen Landesverfassungen ${ }^{1}$, die zwischen November 1946 und Dezember 1947 verabschiedet wurden, direktdemokratische Verfahren vorsahen. Auch unter den sechs nach dem Grundgesetz in Kraft getretenen Verfassungen befanden sich drei, die plebiszitäre Elemente enthielten; lediglich Schleswig-Holstein, Niedersachsen und Hamburg waren damals dem Vorbild des Bundes gefolgt und hatten auf die Einführung der Plebiszite vollständig verzichtet.

In der Praxis spielten die direktdemokratischen Instrumente bis zu Beginn der neunziger Jahre keine große Rolle, wenn man von wenigen spektakulären Fällen absieht. Hierzu gehörte zum Beispiel das Volksbegehren gegen die von der Landesregierung geplante Einführung der Kooperativen Schule in Nordrhein-Westfalen, das 1978 auch bundesweit Schlagzeilen machte. Die Gründe für die Zurückhaltung lagen zum einen in der restriktiven Ausgestaltung der Volksgesetzgebung, zum anderen im anti-plebiszitären Zeitgeist und dem wachsenden Grundvertrauen in die repräsentativen Institutionen. Dementsprechend gab es auch keine Ambitionen, an der Verfassungslage etwas zu ändern. Die einzige Ausnahme blieb lange Zeit Baden-Württemberg, das sich 1974 zur Einführung einer zweistufigen Volksgesetzgebung entschloss, nachdem es Bürgerbegehren und -entscheid 18 Jahre zuvor bereits auf der kommunalen Ebene eingerichtet hatte. Umgekehrt entschied sich das Land Berlin im selben Jahr dafür, aus der praktischen Folgenlosigkeit des Instruments die Konsequenzen zu ziehen, indem es bis auf die plebiszitäre Parlamentsauflösung alle Volksrechte abschaffte. $^{2}$

\section{Der Ausbau der Direktdemokratie in Ländern und Kommunen}

Richtige Schubkraft sollte die plebiszitäre Entwicklung erst gegen Ende der achtziger Jahre entfalten. Ursächlich dafür waren eine Reihe von Faktoren, deren zeitnahes Zusammentreffen die Reformen beflügelte. Der Novellierungsbedarf der vorgrundgesetzlichen Landesver-

* Für nützliche Hinweise danke ich Marcel Solar.

1 Darin eingeschlossen sind die fünf Länder der damaligen Sowjetischen Besatzungszone und die Länder Baden, Württemberg-Baden und Württemberg-Hohenzollern, die sich 1952 zum Land Baden-Württemberg vereinigten.

2 Vgl. Bärbel Martina Weixner, Direkte Demokratie in den Bundesländern. Verfassungsrechtlicher und empirischer Befund aus politikwissenschaftlicher Sicht, Opladen 2002. 
fassungen und wenig später die Verfassungsgebung in den neuen Bundesländern schufen günstige Gelegenheiten für die Einführung der Plebiszite, die durch das allgemein gestiegene Bedürfnis nach politischer Partizipation und die demokratische Aufbruchstimmung in der früheren DDR zusätzlich unterstützt wurden. ${ }^{3}$ Vorreiter der Reform war das von der Barschel-Affäre geschüttelte Land Schleswig-Holstein, dessen 1990 verabschiedete neue Verfassung für die anderen Länder Modellcharakter gewann. Die Dynamik des anschließenden Prozesses lässt sich einerseits mit der Attraktivität des Themas im Parteienwettbewerb, andererseits mit dem föderalen Nachahmungsdruck erklären. Dies führte zumal auf der kommunalen Ebene zu einem regelrechten Dominoeffekt, durch den der bis dahin nur in Baden-Württemberg gekannte Bürgerentscheid ab 1990 in allen Kommunalverfassungen Einzug hielt. Begleitet wurde dieser Prozess von der flächendeckenden Einführung der Direktwahl der Bürgermeister, die vor 1992 - im Rahmen der Süddeutschen Ratsverfassung - ebenfalls nur in Bayern und Baden-Württemberg vorgesehen war. ${ }^{4}$ Begünstigend wirkte sich auch die direkte Demokratie selbst aus, die - einmal eingeführt - als Hebel für weitere Verfassungsänderungen diente. So konnte auf der Länderebene schon 1996 der förmliche Schlussstein unter den Reformprozess gesetzt werden, als Hamburg als letztes Bundesland direktdemokratische Instrumente in seine Verfassung übernahm.

\section{Anwendungshäufigkeit und-erfahrungen in der Praxis}

Ein wesentlich nüchterneres Bild ergibt sich, wenn man die tatsächliche Anwendungspraxis betrachtet. Zwar lässt sich auf der Länderebene ein deutlicher Anstieg der eingeleiteten Begehren und durchgeführten Volksentscheide verzeichnen, seitdem das direktdemokratische Instrumentarium ausgeweitet wurde, doch bewegt sich die Gesamtzahl nach wie vor auf sehr niedrigem Niveau. Der Befund wird durch die höchst ungleiche Verteilung zwischen den Ländern unterstrichen. Im gesamten Zeitraum von 1947 bis 2008 wurden gerade einmal 15 Volksentscheide durchgeführt, von denen allein zehn auf zwei Bundesländer - Bayern und Hamburg - entfielen. ${ }^{5}$ In zehn Ländern fand bisher überhaupt kein Volksentscheid statt. Ähnlich sieht die Verteilung bei den Volksbegehren aus. Deren Gesamtzahl beläuft sich auf 61, von denen wiederum mehr als die Hälfte (34) in nur drei Ländern Bayern, Brandenburg und Hamburg - stattfanden. Baden-Württemberg, MecklenburgVorpommern und das Saarland verzeichnen bislang kein einziges Volksbegehren, Hessen und Rheinland-Pfalz, wo das Instrument schon seit 1946 beziehungsweise 1947 besteht, nur jeweils eines. ${ }^{6}$

3 Vgl. Frank Decker, Systemrezeption und institutionelle Innovationen im deutschen Einigungsprozess. Eine Bilanz, in: ZPol, 14. Jg. (2004), H. 1, S. $31-67$.

4 Vgl. Hans Herbert von Arnim, Vom schönen Schein der Demokratie. Politik ohne Verantwortung - am Volk vorbei, München 2000.

5 Mit den Abstimmungen über den Nichtraucherschutz in Bayern und die Schulreform in Hamburg gesellten sich 2010 zwei weitere Volksentscheide hinzu.

6 Vgl. Frank Rehmet, Volksbegehrensbericht 2008, Mehr Demokratie e.V., Berlin 2009. 


\begin{tabular}{|c|c|c|c|c|c|}
\hline Bundesland & $\begin{array}{l}\text { Einführung } \\
\text { der Plebiszite }\end{array}$ & $\begin{array}{c}\text { Volks- } \\
\text { initiativen* }\end{array}$ & $\begin{array}{c}\text { Volks- } \\
\text { begehren }\end{array}$ & $\begin{array}{c}\text { Volks- } \\
\text { entscheide }\end{array}$ & $\begin{array}{l}\text { Verfassungs- } \\
\text { referenden }\end{array}$ \\
\hline Baden-Württemberg & 1974 & 5 & - & - & 3 \\
\hline Bayern & 1946 & 41 & 16 & 5 & 10 \\
\hline Berlin & $\begin{array}{c}1949-1974 \\
1995\end{array}$ & 18 & 3 & 1 & 3 \\
\hline Brandenburg & 1992 & 26 & 8 & - & 2 \\
\hline Bremen & 1947 & 10 & 4 & - & 3 \\
\hline Hamburg & 1996 & 25 & 10 & 5 & - \\
\hline Hessen & 1946 & 6 & 1 & - & 10 \\
\hline Mecklenburg-Vorpommern & 1994 & 21 & - & - & 1 \\
\hline Niedersachsen & 1993 & 7 & 2 & - & - \\
\hline Nordrhein-Westfalen & 1950 & 11 & 2 & - & 1 \\
\hline Rheinland-Pfalz & 1947 & 5 & 1 & - & 2 \\
\hline Saarland & 1979 & 6 & - & - & - \\
\hline Sachsen & 1992 & 11 & 4 & 1 & - \\
\hline Sachsen-Anhalt & 1992 & 3 & 2 & 1 & - \\
\hline Schleswig-Holstein & 1990 & 20 & 4 & 2 & - \\
\hline Thüringen & 1994 & 7 & 4 & - & 1 \\
\hline Summe & & 222 & 61 & 15 & 36 \\
\hline
\end{tabular}

Auf der kommunalen Ebene liegt die Zahl der Bürgerbegehren und -entscheide naturgemäß höher. Nach Erhebungen des Vereins „Mehr Demokratie“ beläuft sie sich auf 3.721 beziehungsweise 2.226. (Von den Entscheiden geht dabei knapp ein Drittel auf ein Ratsbegehren zurück.) Die Verteilung ist auch hier sehr unterschiedlich: Allein Bayern kommt auf rund 40 Prozent aller Verfahren und Entscheide. Betrachtet man die Kommunen im einzelnen, liegt München mit 20 Verfahren an der Spitze, gefolgt vom Hamburger Bezirk Wandsbek (19) und Augsburg (18). Die meisten Bürgerentscheide fanden in Erlangen (13) und Regensburg (10) statt. Laut „Mehr Demokratie“ hat es in einem Fünftel der deutschen Städte und Gemeinden bisher mindestens ein Verfahren gegeben. Im Umkehrschluss heißt das, dass 80 Prozent der Kommunen noch keine Bekanntschaft mit dem direktdemokratischen Instrumentarium gemacht haben. ${ }^{7}$

Dass die direkte Demokratie im realen Verfassungsleben nur eine geringe Rolle spielt, hängt vor allem mit ihrer restriktiven Ausgestaltung zusammen. Ursächlich dafür sind zum einen die Regelungen selbst, zum anderen ihre überwiegend defensive Interpretation durch die Verfassungsrechtsprechung. ${ }^{8}$ Alle Bundesländer haben sich für die vermeintlich progres-

7 Vgl. Frank Rehmet / Volker Mittendorf, Erster Bürgerbegehrensbericht 1956 - 2007, erstellt von Mehr Demokratie e.V. in Kooperation mit der Forschungsstelle Bürgerbeteiligung und Direkte Demokratie der Universität Marburg, Berlin 2008.

8 Vgl. Peter Neumann, Die Entwicklung der Rechtsprechung zu Volksbegehren und Volksentscheid nach der Deutschen Einheit, in: Theo Schiller / Volker Mittendorf(Hrsg.), Direkte Demokratie, Wiesbaden 2002, S. $115-152$. 
sivste Variante der Direktdemokratie entschieden, die so genannte Volksgesetzgebung. Diese gibt den Bürgern die Möglichkeit, selbst als Gesetzgeber aktiv zu werden und nötigenfalls an die Stelle - nicht nur an die Seite - des parlamentarischen Gesetzgebers zu treten. Damit unterscheidet sie sich grundsätzlich von anderen Formen der Abstimmung, die „von oben“ anberaumt werden (einfaches Referendum), durch die Verfassung vorgeschrieben sind (obligatorisches Referendum) oder sich auf ein bereits beschlossenes Gesetz beziehen (Vetoinitiative beziehungsweise fakultatives Referendum).

Die meisten Bundesländer kennen die Volksgesetzgebung in der dreistufigen Abfolge von Initiative, Begehren und Entscheid. Baden-Württemberg, Bayern, Hessen und das Saarland praktizieren ein zweistufiges Verfahren, das ohne die Initiative auskommt; dasselbe Verfahren gilt in sämtlichen Kommunalverfassungen. Bemerkenswert ist, dass mit der Entscheidung für das Volksgesetzgebungsmodell ein weitgehender Verzicht auf die anderen Beteiligungsformen einhergeht. ${ }^{9}$ Ein obligatorisches Verfassungsreferendum ist nur in Bayern und Hessen gegeben. In vier Ländern (Baden-Württemberg, Bremen, Nordrhein-Westfalen und Sachsen) kann ein Verfassungsreferendum von Parlament oder Regierung angesetzt werden, wenn ein verfassungsänderndes Gesetz im parlamentarischen Verfahren scheitert. Erwähnenswert ist auch, dass immerhin sieben Länder - Baden-Württemberg, Bayern, Berlin, Brandenburg, Bremen, Nordrhein-Westfalen (dort allerdings nur mittelbar) und Rheinland-Pfalz - zusätzlich zu der in allen Landesverfassungen vorgesehenen Selbstauflösung eine plebiszitäre Abberufung des Parlaments gestatten. Ein einfaches Gesetz zur Abstimmung zu stellen ist dagegen außer in Baden-Württemberg, Nordrhein-Westfalen und Rheinland-Pfalz nirgendwo möglich; dasselbe gilt für die Initiative gegen ein bereits beschlossenes Gesetz, die in abgeschwächter Form nur in Rheinland-Pfalz vorgesehen ist. ${ }^{10}$

Während die Landes- und Kommunalverfassungen in der Grundausrichtung der Direktdemokratie übereinstimmen, bestehen deutliche Unterschiede bei der Ausgestaltung der Verfahren im einzelnen. Empirische Untersuchungen bestätigen die Vorahnung, dass die Nutzungshäufigkeit der Regelungen mit ihrer Nutzerfreundlichkeit korrespondiert: Je größer die Offenheit der Volksgesetzgebung, desto mehr Verfahren werden initiiert und haben die Chance, zum Erfolg zu kommen. ${ }^{11}$

Ihre bedeutendste Einschränkung erfährt die Direktdemokratie durch die Ausschlussgegenstände. Im Anschluss an die Weimarer Reichsverfassung, die eine entsprechende Regelung in Art. 73 vorsah, nehmen sämtliche Landesverfassungen die so genannte Finanztrias von Haushaltsgesetz, Abgaben und Besoldung von der Volksgesetzgebung aus. Bezogen auf

9 Davon ausgenommen ist lediglich die unverbindliche Volkspetition, die unter verschiedenen Bezeichnungen - Volkspetition, Volksinitiative oder Bürgerantrag - in neun Bundesländern neben der Volksgesetzgebung besteht.

10 Vgl. Johannes Rux, Direkte Demokratie in Deutschland. Rechtsgrundlagen und Rechtswirklichkeit der unmittelbaren Demokratie in der Bundesrepublik Deutschland und ihren Ländern, Baden-Baden 2008. „Abgeschwächt" deshalb, weil die Antragsberechtigung nicht beim Volk, sondern bei einem Drittel des Landtags liegt (Art. 114 Satz 1 und Art. 115 Abs. 1 Satz 1 der rheinland-pfälzischen Verfassung). Die hamburgische Verfassung sieht nach der jüngsten Reform der Volksgesetzgebung die Möglichkeit eines (von 2,5 Prozent der Wahlberechtigten zu beantragenden) fakultativen Referendums vor, wenn das Parlament ein volksbeschlossenes Gesetz abändert (siehe unten).

11 Vgl. Christina Eder / Raphael Magin, Direkte Demokratie, in: Markus Freitag / Adrian Vatter (Hrsg.), Die Demokratien der Bundesländer, Opladen / Farmington Hills 2008, S. 257 - 308. 
das Haushaltsgesetz hat das zur Folge, dass auch finanzwirksame Gesetze dem plebiszitären Zugriff weitgehend entzogen sind. Des Weiteren ist ausgeschlossen, dass Verwaltungsentscheidungen und der Landesvollzug von Bundesgesetzen zum Gegenstand eines Volksgesetzgebungsverfahrens gemacht werden, obwohl im deutschen Föderalismus gerade hier die Hauptaufgabe der Länder liegt. ${ }^{12}$ Dieser Ausschluss fällt umso schwerer ins Gewicht, als deren Gesetzgebungskompetenzen im Vergleich zu jenen des Bundes ohnehin sehr schmal geschnitten sind.

Weichen die Verfassungen bei der Festlegung der Ausschlussgegenstände kaum voneinander ab, so offenbart ein Vergleich der in den verschiedenen Stadien des Verfahrens vorgeschriebenen Quoren interessante Unterschiede. Die neuen Bundesländer sowie diejenigen Länder der Altbundesrepublik, die ihre Verfassungen erst in jüngerer Zeit novelliert haben (Niedersachsen und die Stadtstaaten), sind bei der Ausgestaltung der Volksgesetzgebung dem so genannten „Kieler Modell“ gefolgt, das geringe Beteiligungshürden und großzügig bemessene Eintragungsfristen in der Eingangsphase mit einem vergleichsweise hohen $\mathrm{Zu}$ stimmungsquorum kombiniert (zwischen 20 und 33 Prozent). Bayern, Hessen, Sachsen und - mit Abstrichen - Nordrhein-Westfalen haben den umgekehrten Weg beschritten: hohe Einstiegshürden und kurze Eintragungsfristen bei Initiative und Begehren, dafür aber ein niedrigeres beziehungsweise überhaupt kein Zustimmungsquorum beim Entscheid. ${ }^{13}$ Bildet man eine Rangfolge, stehen Bayern, Hamburg und Sachsen als Länder mit den niedrigsten Schwellen an der Spitze, während das Saarland mit einem in der Praxis unerreichbaren Zustimmungsquorum von 50 Prozent (!) am Ende rangiert. ${ }^{14}$

In engem Zusammenhang mit den Quoren stehen die Fristen, die für das Zustandekommen eines Volksbegehrens einzuhalten sind, sowie die Modalitäten der Unterschriftensammlung. Die Fristen reichen von lediglich 14 Tagen (Baden-Württemberg, Bayern, Hessen und Saarland) bis zu gar keiner Frist (Mecklenburg-Vorpommern). ${ }^{15}$ Fasst man Quoren und Fristen zu einem Mobilisierungskoeffizienten zusammen, ist es in Hessen fünfzig Mal schwerer, die Volksbegehrenshürde zu nehmen, als in Schleswig-Holstein. ${ }^{16}$ Abschreckende Wirkung hat es auch, wenn die Unterschriften für ein Begehren in der Amtsstube zu leisten sind und nicht (wie in der Initiativphase) frei gesammelt werden können. Dies ist zurzeit in acht Ländern vorgeschrieben; die restlichen acht erlauben die freie Unterschriftensammlung.

Weitere Restriktionen ergeben sich aus den Regeln der Kostenübernahme. Zwar sehen alle Länder vor, dass die Initiatoren die Kosten in der Eingangsphase und beim Volksbegehren zunächst selbst tragen müssen. In einigen Ländern können sie jedoch für die Aufwendungen entschädigt werden - sei es durch eine Erstattung der tatsächlich entstandenen Kosten oder durch eine Vergütung, die sich analog zur Wahlkampfkostenerstattung an der Unterstützung des Begehrens beziehungsweise Abstimmungsbeteiligung orientiert. ${ }^{17}$

12 Vgl. Bärbel Martina Weixner, a.a.O. (Fn. 2), S. 98 f.

13 In Sachsen-Anhalt entfällt das Zustimmungsquorum, wenn der Landtag eine Konkurrenzvorlage zur Abstimmung stellt.

14 Vgl. Frank Rehmet, a.a.O. (Fn. 6), S. 7.

15 Das gilt allerdings nur für den Fall, dass die Unterschriften frei gesammelt werden. Bei der Amtseintragung ist die Frist auf zwei Monate begrenzt.

16 Vgl. Otmar Jung, Grundsatzfragen der direkten Demokratie, in: Andreas Kost (Hrsg.), Direkte Demokratie in den deutschen Ländern, Wiesbaden 2005, S. 312 - 357.

17 Vgl. Christina Eder / Raphael Magin, a.a.O. (Fn. 11), S. 257 - 308. 
Von erheblicher Bedeutung ist schließlich die Terminierung des abschließenden Entscheids. Findet dieser parallel zu einer regulären Wahl statt, ist die Wahrscheinlichkeit, dass das Beteiligungs- oder Zustimmungsquorum überschritten wird, sehr viel größer als bei einer getrennt anberaumten Abstimmung. Nur in Hamburg schreibt die Verfassung zwingend vor, dass Volksentscheide zeitgleich mit der Bundestags- oder Bürgerschaftswahl abzuhalten sind - es sei denn, die Initiatoren selbst beantragen einen anderen Termin. Diese Regelung hat eine interessante Geschichte. Nachdem sie 2004 bei gleich zwei Volksabstimmungen empfindliche Niederlagen erlitten hatte, setzte die regierende CDU eine Änderung des Ausführungsgesetzes durch, die die Koppelung der Abstimmungen an die Wahlen beseitigte. ${ }^{18}$ Das Hamburgische Verfassungsgericht hob den Beschluss auf, da die Verfassung die gleichzeitige Durchführung von Wahlen und Abstimmungen ausdrücklich gestatte. Die Regierungspartei nahm dies zum Anlass, auf die Initiatoren zuzugehen und einen für beide Seiten akzeptablen Kompromiss zu suchen. Die im Dezember 2008 von der Bürgerschaft verabschiedete Verfassungsänderung sieht nun unterschiedliche Quoren vor, je nachdem, ob die Abstimmungen zeitgleich mit der Wahl stattfinden oder nicht: Im ersten Falle müssen mindestens so viele Wähler der Vorlage zustimmen, wie der Mehrheit der in dem gleichzeitig gewählten Parlament repräsentierten Hamburger Stimmen entspricht, in letzterem Falle genügen 20 Prozent der Wahlberechtigten. ${ }^{19}$

Ohne bei der schier unendlichen Regelungsvielfalt noch weiter ins Detail zu gehen, lassen sich die vorstehenden Ausführungen zur Ausgestaltung der Direktdemokratie auf der kommunalen und Landesebene in drei Feststellungen zusammenfassen: Erstens bleiben die Anwendungsbedingungen in der Summe so restriktiv, dass die plebiszitären Elemente in der Verfassungspraxis nur eine geringe Rolle spielen und eine nachhaltige, systemverändernde Wirkung von ihnen nicht ausgeht. Die benutzerfreundlichsten Länder in der Bundesrepublik bewegen sich ungefähr auf demselben Niveau wie die benutzerunfreundlichsten Schweizer Kantone oder US-Bundesstaaten. Zweitens bestehen in den Anwendungsbedingungen enorme Unterschiede zwischen den Ländern, die sich in der tatsächlichen Nutzung der Verfahren widerspiegeln. In keinem anderen Bereich der Landesregierungssysteme ist die institutionelle Bandbreite so groß wie hier. Drittens konnten die Anwendungsbedingungen nach der Einführung der Direktdemokratie im Schnitt verbessert werden. Weil auch hier nur ein Teil der Länder aktiv voranging und die anderen den Trend mehr oder weniger verschliefen, hat sich die Schere zwischen den Vorreitern und Nachzüglern seither nochmals vergrößert.

18 Vgl. Andreas von Arnauld, „Refolution“ an der Elbe: Hamburgs neue direkte Demokratie - Die Verfassungsänderungen der Jahre 2008 und 2009 im Kontext, in: Lars P. Feld / Peter M. Huber / Otmar Jung / Christian Welzel / Fabian Wittreck (Hrsg.), Jahrbuch für direkte Demokratie 2009, Baden-Baden 2010, S. 90 - 130, S. 98.

19 Vgl. Frank Rehmet, a.a.O. (Fn. 6), S. 26. Die Regelung knüpft an Art. 2 Sect. 8 (b) und 9 (b) der kalifornischen Verfassung an, der eine entsprechende Koppelung an die Gouverneurswahlen vorsieht. Demokratiepolitisch inkonsequent ist sie im Hamburger Fall insofern, als bei einer Bezugnahme auf die Bundestagswahl der systemische Zusammenhang zwischen parlamentarischer und Volksgesetzgebung auf Landesebene durchbrochen wird. Das zeigt, dass es den Initiatoren weniger um eine Synchronisierung des Legitimationsniveaus der beiden konkurrierenden Landesgesetzgebungsorgane ging, als darum, die Beteiligungsraten bei den Volksabstimmungen zu erhöhen. Vgl. Andreas von Arnauld, a.a.O. (Fn. 18), S. $111 \mathrm{ff}$. 
Dass die direkte Demokratie selbst einen wesentlichen Beitrag geleistet hat, um diese Entwicklung zu ermöglichen, ist hierbei mehr als nur eine ironische Fußnote. Schlüsselt man die 266 Volksinitiativen, -petitionen und -begehren, die laut Auskunft von „Mehr Demokratie“ von 1946 bis 2008 lanciert wurden, nach Themengebieten auf, liegt der Bereich „Demokratie, Staatsorganisation und Innenpolitik“ mit 21 Prozent gleich an zweiter Stelle - nach „Bildung und Kultur“ mit 29 Prozent. ${ }^{20}$ Etwa die Hälfte davon entfallen wiederum allein auf die direkte Demokratie. Nimmt man nur den Zeitraum seit 1991, ab dem die plebiszitären Elemente flächendeckend eingerichtet wurden beziehungsweise Bestand hatten, liegt der Anteil sogar bei zwei Dritteln. ${ }^{21}$ Aus den Zahlen spricht einerseits das Bedürfnis vieler Wähler und Aktivisten, die als zu restriktiv empfundenen Anwendungsbedingungen der Plebiszite zu lockern, zum anderen - wie gesehen - der weitreichende Ausschluss sonstiger abstimmungsfähiger Themen. Auch von den Initiativen zur Direktdemokratie sind die meisten schon vorab als unzulässig erklärt worden. ${ }^{22}$ Nur in vier Fällen (einer in Bayern und drei in Hamburg) gelang es, einen Volksentscheid zu erzwingen. Zwei davon waren erfolgreich - der Entscheid über die Einführung des kommunalen Bürgerentscheids in Bayern (1995), bei dem sich der Entwurf von „Mehr Demokratie“ gegen die Konkurrenzvorlage des Bayerischen Landtags durchsetzen konnte, und der Entscheid über die Einführung des Bürgerentscheids in den Hamburger Bezirken (1998).

Der Erfolg der Direktdemokratie in eigener Sache lässt sich mit diesen Zahlen aber nicht annähernd erfassen, denn auch wo die Vorstöße über das Initiativ- oder Begehrensstadium nicht hinauskamen, wurden sie von den Parlamenten in der Regel aufgegriffen. Auf diese Weise konnten zum Beispiel in Berlin, Bremen und Thüringen teilweise erhebliche Verbesserungen erreicht werden. Besonders spektakulär war die Wirkung der beiden „unecht" (das heißt lediglich am Zustimmungsquorum) gescheiterten Initiativen für erleichterte Volksbegehren und -entscheide in Hamburg (1998 und 2007), denen eine klare Mehrheit der Wähler zugestimmt hatte. Dies wurde von den Regierenden als Aufforderung verstanden, die Volksgesetzgebung grundlegend zu reformieren. Dass das ehemalige Schlusslicht Hamburg heute unter allen Bundesländern die anwenderfreundlichsten Verfahren hat, verdankt sich also ganz unmittelbar der direkten Demokratie. ${ }^{23}$

Die rasche Folge der mit Hilfe des Plebiszits durchgesetzten Verbesserungen ist mehreren Umständen geschuldet. Zum einen haben sich viele Initiativen nicht auf die förmliche Verfassung bezogen, wo die Änderungshürden sehr hoch liegen, sondern auf die leichter veränderbaren Ausführungsgesetze. Zum anderen waren ihnen oftmals Versuche der Verfassungsgerichte oder des Gesetzgebers vorausgegangen, den erreichten Zustand zurückzudrehen und die Anwendbarkeit der direktdemokratischen Verfahren zu beschneiden. Dass die pro-plebiszitären Kräfte in diesem Hase-Igel-Rennen am Ende meistens die Nase vorn hatten, mag man im Sinne einer dynamischen Verfassungsentwicklung begrüßen. Zur Idee der Verfassung gehört allerdings auch, dass sie auf eine gewisse Dauerhaftigkeit hin angelegt ist. Ständiger Streit um einen wichtigen Teil seiner konstitutionellen Grundlagen tut auf lange Sicht keinem Gemeinwesen gut. Das Hin und Her zwischen plebiszitfreundlicher und

20 Vgl. Frank Rehmet, a.a.O. (Fn. 6). S. 12 ff.

21 Vgl. die Aufstellung bei Johannes Rux, a.a.O. (Fn. 10), S. $931 \mathrm{ff.}$

22 Vgl. Otmar Jung, a.a.O. (Fn. 16), S. $312 \mathrm{ff.}$

$23 \mathrm{Vgl}$. Andreas von Arnault, a.a.O. (Fn. 18), S. 94 ff. 
restriktiver Ausgestaltung, das die Volksgesetzgebung provoziert, erweist sich unter diesem Gesichtspunkt als ernsthaftes Problem.

\section{Volksgesetzgebung und parlamentarisches System}

Aus normativer Sicht spricht vieles dafür, die (positive) Initiative, die den Kern des Volksgesetzgebungsmodells ausmacht, als vorzugswürdigste Form der Direktdemokratie zu betrachten. Bei einem Referendum liegt es im Ermessen der Regierenden, ob sie das Volk entscheiden lassen, bleibt die Direktdemokratie also ein Instrument der „Politik von oben“. Im Rahmen der Volksgesetzgebung erfolgt der plebiszitäre Impuls demgegenüber „von unten “. Hier können die Bürger selber in Aktion treten und sich als Gesetzgeber an die Stelle der repräsentativen Institutionen setzen. Auf den ersten Blick scheint es sympathisch, dass Länder und Kommunen in der Bundesrepublik sich sämtlich diesem fortschrittlichen Modell verschrieben haben. Von der positiven Bewertung bleibt bei genauerer Betrachtung allerdings nicht viel übrig. Einerseits zeigt sich, dass die erschwerte Anwendbarkeit der plebiszitären Verfahren eine unmittelbare Folge der Entscheidung für das Volksgesetzgebungsmodell darstellt: Gerade weil man den Bürgern die potenziell weitreichendsten Mitwirkungsmöglichkeiten eröffnet, sieht man sich gezwungen, diese in der Praxis sogleich wieder zu beschränken. Andererseits provoziert die Volksgesetzgebung Konflikte, falls es den Initiatoren doch einmal gelingen sollte, die Hürden zu überspringen und sich gegen den Willen des parlamentarischen Gesetzgebers zu stellen. Die positive Initiative wirft mit anderen Worten Vereinbarkeitsprobleme mit dem herkömmlichen parlamentarischen Entscheidungsverfahren auf, die in der Forschung bislang systematisch wenig angesprochen wurden.

Die Skepsis verstärkt sich noch, wenn man die Bundesrepublik mit anderen Ländern vergleicht. Gewiss ist es nicht möglich, Strukturen, die historisch gewachsen und in einen bestimmten Systemkontext eingebettet sind, ohne Weiteres in einen anderen Kontext zu übertragen: „Einen Rhododendronbusch aus Bergen in Norwegen sollte man nicht in den Havelsand verpflanzen, und das Edelweiß aus den Schweizer Alpen würde in der Bundesrepublik Deutschland jämmerlich eingehen “24. Der Ländervergleich zeigt aber auch, dass die institutionellen Merkmale eines politischen Systems keineswegs beliebig zusammenwirken - manche Kombinationen sind „funktionaler“ als andere. Insofern ist es geradezu geboten, die ausländischen Vorbilder im Rahmen von Verfassungsgebungsprozessen gründlich zu studieren. Dies gilt insbesondere dann, wenn es sich - wie bei der Direktdemokratie - um „optionale" Einrichtungen handelt, die nicht zum institutionellen Mindestbestand der Verfassung gehören (wie Parlamentarismus, Wahlrecht, Verfassungsgerichtsbarkeit oder der territoriale Staatsaufbau).

Der europaweite Vergleich ergibt hier zunächst einen überraschenden Befund. Während die Möglichkeit eines von oben anzuberaumenden Referendums in unterschiedlichen Varianten fast überall gegeben ist, gilt dies nicht für die Volksinitiative. Lässt man Zwergstaaten wie Liechtenstein oder San Marino außer Betracht, räumen in Europa lediglich fünf parlamentarische Demokratien - Italien, Litauen, die Slowakei, Slowenien und Ungarn - den

24 Manfred G. Schmidt, Lehren der Schweizer Referendumsdemokratie, in: Claus Offe (Hrsg.), Demokratisierung der Demokratie, Frankfurt am Main / New York 2003, S. 111 - 123. 
Bürgern das Recht ein, ein vom Parlament beschlossenes Gesetz mittels Vetoinitiative aufzuheben. Litauen, die Slowakei und Ungarn gestehen dem Volk darüber hinaus ein positives Gesetzgebungsrecht zu, das sich allerdings nicht auf alle Materien erstreckt. Von Italien abgesehen ist die Volksinitiative auf der nationalen Ebene also nur in einigen mittel- und osteuropäischen Ländern verbreitet, wo man sie nach dem Systemumbruch 1989 aus einem demokratischen Überschwang heraus eingeführt hat. ${ }^{25}$

Unter den präsidentiellen Systemen ist das Bild gemischter. Während sich die US-amerikanische Bundesverfassung bis heute in plebiszitärer Enthaltsamkeit übt, ist die Initiative - in Kombination mit dem Referendum - mittlerweile in den meisten Staaten Lateinamerikas geläufig. ${ }^{26}$ Auf der anderen Seite sieht in den USA immerhin rund die Hälfte der Einzelstaaten zum Teil sehr weitreichende Initiativrechte vor. ${ }^{27}$ Dasselbe gilt für die präsidentiell (auf der gliedstaatlichen Ebene) beziehungsweise „quasi-präsidentiell“ (auf der nationalen Ebene) verfasste Schweiz - das Land mit der am stärksten ausgebauten Direktdemokratie in der Welt. Hier gibt es die Initiative auf der nationalen Ebene bis heute zwar nur als Verfassungsinitiative - die direktdemokratischen Verfahren konzentrieren sich nicht in der Volksgesetzgebung, sondern in der Vetoiniative gegen ein bereits beschlossenes Gesetz, also dem fakultativen Referendum. ${ }^{28}$ Unter dem Strich bleibt damit aber das Ergebnis, dass beide Formen - die positive wie die negative Initiative - in einem parlamentarischen Regierungssystem offenbar schlechter aufgehoben sind als in einem präsidentiellen. Die Frage nach den Gründen lässt sich in einen normativ-verfassungsrechtlichen und einen institutionell-funktionalen Aspekt unterteilen.

Aus normativ-verfassungsrechtlicher Sicht geht es um die Verbindlichkeit der volksbeschlossenen Gesetze. Befürworter und Gegner der Plebiszite stimmen darin überein, dass Volks- und Parlamentsgesetze als prinzipiell gleichrangig betrachtet werden müssen. Beide wären damit wechselseitig aufhebungsfähig. Das heißt: So wie das Volk die Möglichkeit hat, ein Parlamentsgesetz einem Referendum zu unterwerfen oder durch eine spätere (positive) Gesetzesinitiative zu korrigieren, so muss auch das Parlament ein vom Volk beschlossenes Gesetz wieder rückgängig machen können. ${ }^{29}$ Dennoch wäre es falsch, von einer vollständigen Symmetrie der beiden Verfahrenstypen auszugehen. Dazu muss man keine normative Höherwertigkeit der Plebiszite unterstellen, wie es pro-direktdemokratisch argumentierende Autoren wie Hans Herbert von Arnim oder Otmar Jung ${ }^{30}$ tun. Eine solche Höherwertigkeit kommt ihnen im Sinne einer Bindewirkung allenfalls politisch zu. Einerseits wird nur einer kleiner Teil zumeist besonders wichtiger Gesetze auf plebiszitärem Wege beschlossen. Andererseits würde das Vertrauen der Bürger in die Direktdemokratie unter-

25 Vgl. Benjamin Ewert, Potentiale der direkten Demokratie in Litauen, Slowenien und Ungarn unter besonderer Berücksichtigung der politischen Kultur, Frankfurt am Main 2007.

26 Vgl. Stefan Rinke / Klaus Stüwe, Politische Systeme Amerikas: Ein Vergleich, in: Klaus Stüwe I Stefan Rinke (Hrsg.), Die politischen Systeme in Nord- und Lateinamerika, Wiesbaden 2008, S. $9-58$.

27 Vgl. Silvano Moeckli, Direkte Demokratie in den Gliedstaaten der USA, in: Markus Freitag / Uwe Wagschal (Hrsg.), Direkte Demokratie, Berlin 2007, S. 19 - 39.

28 Vgl. ders., Direktdemokratische Einrichtungen und Verfahren in den Mitgliedstaaten des Europarates, in: ZParl, 29. Jg. (1998), H. 1, S. $90-107$.

29 Vgl. Martin Borowski, Parlamentsgesetzliche Änderungen volksbeschlossener Gesetze, in: DÖV, 53. Jg. (2000), H. 12, S. $481-491$.

30 Vgl. Otmar Jung, a.a.O. (Fn. 16). S. 334. 
graben, wenn das Parlament einen Volksentscheid schon am nächsten Tage wieder aufhöbe. Deshalb ist es durchaus zweckmäßig, den volksbeschlossenen Gesetzen einen höheren Bestandsschutz einzuräumen als den Parlamentsgesetzen. Eine Lösung könnte beispielsweise darin liegen, dass man bestimmte Fristen vorsieht, innerhalb derer der parlamentarische Gesetzgeber auf ein plebiszitär zustande gekommenes Gesetz nicht zugreifen darf. Noch konsequenter wäre es, die Aufhebung oder Korrektur eines solchen Gesetzes von einem neuerlichen Volksvotum abhängig zu machen ${ }^{31}$ oder sie der Möglichkeit einer Vetoinitiative zu unterwerfen, wie es die in diesem Punkt geänderte Hamburgische Verfassung in Art. 50 Abs. 4 und 4 a jetzt vorsieht. ${ }^{32}$ Die Neuregelung kam auf Druck eines von „Mehr Demokratie" lancierten Volksbegehrens zustande, das neben anderen Verbesserungen auch eine größere Verbindlichkeit der Volksentscheide anstrebte. Dem waren mehrere Fälle vorausgegangen, in denen der Senat beziehungsweise die Bürgerschaft der Hansestadt Volksbeschlüsse missachtet oder gegen den Willen der Initiatoren korrigiert hatte. ${ }^{33}$

Der Konflikt zwischen parlamentarischem und Volksgesetzgeber, der in Hamburg fürs erste befriedet erscheint ${ }^{34}$, leitet zur institutionell-funktionalen Problematik über, also zur Frage, ob sich die plebiszitären Verfahren in die vorhandene repräsentative Demokratie überhaupt sinnvoll einfügen lassen. Um dies zu beantworten, muss zum einen nach dem Charakter der repräsentativen Strukturen und der durch sie geprägten Entscheidungslogik des (gesamten) Regierungssystems gefragt werden. Zum anderen ist zu untersuchen, welche Differenzen hinsichtlich ihrer Systemverträglichkeit zwischen den verschiedenen Varianten der direkten Demokratie bestehen.

Im ersten Punkt kann man auf die von Arend Lijphart entwickelte Unterscheidung von Mehrheits- und Konsensdemokratie als zwei Grundtypen demokratischer Regierungssysteme zurückgreifen. ${ }^{35}$ Die Mehrheitsdemokratie stellt eine Abstraktion des britischen Westminster-Modells dar. Dessen Hauptmerkmale sind zum einen die Realisierung des Prinzips der alternierenden Regierung auf der Basis eines gegnerschaftlich ausgerichteten, dualistischen Parteienwettbewerbs, und zum anderen der Umstand, dass Kabinett und Premierminister über weitreichende Handlungsmacht verfügen, da sie im Parlament in der Regel eine große zahlenmäßige Mehrheit hinter sich wissen und ihre Kreise nicht durch Mitregenten oder sonstige verfassungsrechtliche Gegengewichte gestört werden. Die Konsensdemokratie orientiert sich demgegenüber am Prinzip der Machtteilung. Diese kann institutionell auf unterschiedliche Weise realisiert werden. In den so genannten Konkordanzsystemen findet

31 So der neu beschlossene Art. 73 Abs. 2 der Bremischen Verfassung, der daneben auch die Korrektur eines volksbeschlossenen Gesetzes durch die Bürgerschaft mit qualifizierter verfassungsändernder Mehrheit gestattet.

32 Vgl. Otmar Jung, Volksgesetze und parlamentarische Konterlegislatur, in: Klemens H. Schrenk I Markus Soldner (Hrsg.), Analyse demokratischer Regierungssysteme. Festschrift für Wolfgang Ismayr zum 65. Geburtstag, Wiesbaden 2010, S. 427 - 442, S. 436 ff.

33 Vgl. Frank Decker, Parlamentarische Demokratie versus Volksgesetzgebung. Der Streit um ein neues Wahlrecht in Hamburg, in: ZParl, 38. Jg. (2007), H. 1, S. $118-133$.

34 Nachdem die Durchsetzung verbindlicher Volksentscheide auf Drängen der GAL in den schwarzgrünen Koalitionsvertrag aufgenommen wurde, ist es nicht ohne Ironie, dass sich das erste erfolgreiche Volksbegehren seit der Neuregelung ausgerechnet gegen ein zentrales Regierungsprojekt der Grünen richtete, nämlich die vom Senat beschlossene Schulreform. Diese wurde im Volksentscheid am 18. Juli 2010 von den Bürgern zu Fall gebracht.

35 Vgl. Arend Lijphart, Patterns of Democracy. Government Forms and Performance in Thirty-Six Countries, New Haven / London 1999. 
sie bereits auf der parlamentarischen Ebene statt. An die Stelle des bipolaren Wettbewerbs treten hier Verfahren der "gütlichen“ Konfliktregelung, in die nach Möglichkeit alle relevanten Parteien und Gruppen einbezogen werden. Unter den Regierungsformaten dominieren entsprechend zentristische oder Große Koalitionen, die im Parlament über breite Unterstützung verfügen. Beispiele sind Belgien oder die skandinavischen Länder. Stark ausgeprägte Konkordanzstrukturen waren in der Vergangenheit auch in Österreich, den Niederlanden und Italien anzutreffen, deren politische Systeme sich inzwischen jedoch in Richtung Wettbewerb geöffnet haben. Dies gilt in begrenztem Maße selbst für die Schweiz, die mit ihrer Proporzregierung bis heute den Extremtypus der Konkordanzdemokratie verkörpert.

Bei Lijphart finden sich über die systemischen Wirkungen der Plebiszite nur kryptische Äußerungen. Hatte er in der Erstauflage seines Demokratiebuches noch versucht, die direktdemokratischen Verfahren in seine Typologie zu integrieren, so wurde das Kapitel über die Referenden in der Neufassung ganz gestrichen. Lijpharts These, wonach es zwischen der direkten Demokratie und dem majoritären oder konsensuellen Grundcharakter des politischen Systems keinen Zusammenhang gebe, basiert auf einer unzureichenden Operationalisierung der plebiszitären Variable, bei der ausschließlich die Einsatzhäufigkeit des Instruments zugrunde gelegt wird und nicht dessen unterschiedliche Spielarten. ${ }^{36}$ Dabei hätte sich bereits intuitiv erschließen lassen, dass die Rückwirkungen der Initiative auf das Regierungssystem ganz andere sein müssen als jene des obligatorischen oder einfachen Referendums. ${ }^{37}$

Die Frage der Systemverträglichkeit betrifft in erster Linie die Initiative und stellt sich vor allem mit Blick auf die Mehrheitsdemokratien. Wo die Plebiszite als Initiativrechte ausgestaltet sind, stehen sie in einem natürlichen Spannungsverhältnis zum gegnerschaftlichen Parteienwettbewerb. ${ }^{38}$ Die Initiative würde der parlamentarischen Opposition ein Mittel an die Hand geben, von der Regierungsmehrheit beschlossene Gesetze über den Umweg einer Volksabstimmung nachträglich zu Fall zu bringen. Diese Möglichkeit besteht ganz unmittelbar bei der Vetoinitiative; sie wäre aber auch bei der (positiven) Gesetzesinitiative gegeben, die eingebracht werden könnte, um einen Gegenentwurf durchzusetzen. In einer solchen Situation wäre die Regierung gut beraten, etwaige Widerstände gegen ihr Vorhaben schon im Vorwege einzukalkulieren und inhaltlich zu berücksichtigen. Die Volksinitiative wirkt also ihrer Logik nach „konsensuell“, indem sie für eine breitere Interesseninklusion sorgt. Dies kommt auch in den Verfahren selbst zum Tragen, die zahlreiche Querverbindungen zwischen parlamentarischer und Volksgesetzgebung vorsehen. So können die Landesparlamente in der Bundesrepublik zum Beispiel einen Volksentscheid abwenden, wenn sie sich dem Volksbegehren innerhalb einer bestimmten Frist ganz oder in wesentlichen Teilen anschließen. Kommt es zum Volksentscheid, haben sie wiederum die Möglichkeit, einen eigenen Entwurf mit zur Abstimmung zu stellen. Auch dieser wird in der Regel das Anliegen der Initiative zumindest teilweise aufgreifen.

36 Vgl ders., Democratic Political Systems. Types, Cases, Causes and Consequences, in: Journal of Theoretical Politics, 1. Jg. (1989), H. 1, S. 33 - 48.

37 Zur Kritik vgl. Sabine Jung, Lijpharts Demokratietypen und die direkte Demokratie, in: ZPol, 6. Jg. (1996), H. 3, S. $623-645$.

38 Vgl. Peter Graf Kielmansegg, Über direkte Demokratie - sechs Anmerkungen zu einer unbefriedigenden Debatte, in: Uwe Backes / Eckhard Jesse (Hrsg.), Jahrbuch Extremismus \& Demokratie 2006, 18. Jahrgang, Baden-Baden 2007, S. 57 - 80. 
Stellt man die konsensuellen Wirkungen mit in Rechnung, könnte die Volksgesetzgebung den Regierungsparteien unter dem Strich mehr nützen als der Opposition. Bemächtigt sich letztere des Plebiszits, trägt sie ungewollt dazu bei, dass die Regierung eine "gute“ bleibt und sich ihre Wiederwahlchancen erhöhen. Initiativrechte unterminieren insofern das Prinzip der alternierenden Regierung, auf dem der mehrheitsdemokratische Parlamentarismus basiert. In der Schweiz hat dies dazu geführt, dass im Laufe der Zeit alle großen Parteien und Verbände in den Regierungsprozess integriert wurden und ein fest institutionalisiertes Konkordanzsystem ausbildeten. Die lebhaft praktizierte Abstimmungsdemokratie und das nahezu vollständige Fehlen von Parteienkonkurrenz sind hier Seiten ein und derselben Medaille. ${ }^{39}$

Im Umkehrschluss heißt die, dass dort, wo der Wettbewerb nicht funktioniert und das mehrheitsdemokratische Prinzip ins Leere läuft, die Volksgesetzgebung durchaus ein nützliches, bisweilen sogar unverzichtbares Korrektiv bereithalten kann. Beispiele sind die bis zu Beginn der neunziger Jahre „blockierte“ Demokratie Italiens oder die Dauerherrschaft der CSU in Bayern. In ähnliche Richtung weist der Fall einer Großen Koalition. In dieser atypischen parlamentarischen Konstellation ist das zahlenmäßige Übergewicht der Regierung so groß, dass nicht nur die Kontrolle durch die Opposition leidet, sondern auch das Verfassungsgericht und die Länderkammer als „Vetospieler“ weitgehend ausfallen. Plebiszitäre Elemente drängen sich dann als Ersatz förmlich auf. Die Entwicklung der Parteiensysteme in Bund und Ländern, die Große Koalitionen künftig wahrscheinlicher macht, liefert der Forderung nach mehr direkter Demokratie also zusätzliche Gründe.

Strittiger ist, ob direktdemokratische Instrumente auch in einem majoritären System gut aufgehoben wären. Die Antwort hängt davon ab, wie man die konsensuellen Effekte, die von den Initiativrechten vermutlich ausgehen, mit Blick auf die Gesamtarchitektur des Regierungssystems bewertet. In Deutschland ist die Politikwissenschaft in dieser Frage gespalten. Während die einen eine Stärkung der Mehrheitskomponente empfehlen (zum Beispiel durch eine Wahlrechtsreform), ziehen die anderen die Weisheit des Parteienwettbewerbs als Entscheidungsprinzip in Zweifel und denken über Alternativen zum mehrheitsdemokratischen Parlamentarismus nach. Auch international verlaufen die Debatten zum Teil gegenläufig. Wo die Mehrheitslogik dominiert - wie in Großbritannien - redet man einer konsensuellen Umgestaltung des Systems das Wort; wo das Konsensdenken vorherrscht - wie in der Schweiz -, sollen die Konkordanzzwänge zurückgedrängt werden und mehr Wettbewerb Einzug halten. ${ }^{40}$ Noch weitaus kritischer als in der Schweiz steht man der direkten Demokratie mittlerweile in Kalifornien gegenüber. Nachdem die Weigerung der Bürger, Steuererhöhungen und Ausgabenkürzungen per Volksabstimmung zu beschließen, den Staatshaushalt zuletzt massiv aus dem Gleichgewicht gebracht hat, mehren sich die Stimmen, die höhere Hürden für die bisher leicht zugänglichen Verfahren fordern. ${ }^{41}$ Die Funktionalität ist also auch bei der Volksgesetzgebung keine prinzipielle Frage, sondern kann nur unter Hinweis auf die konkrete Ausgestaltung der Verfahren beantwortet werden.

39 Vgl. Wolf Linder, Schweizerische Demokratie. Institutionen - Prozesse - Perspektiven, Bern / Stuttgart / Wien 2005.

40 Vgl. Raimund E. Germann, Aufnahme plebiszitärer Elemente ins deutsche Grundgesetz. Was lehren die Schweizer Erfahrungen?, in: JfP, 3. Jg. (1993), H. 2, S. 219 - 238.

41 Vgl. Stefan Tomik, Der Terminator in der Zwangsjacke, in: FAZ vom 10. Dezember 2009, S. 3. 


\section{Die präsidentielle Regierungsform als Alternative?}

Bleibt die Frage, ob sich die Vereinbarkeitsproblematik auf der Landesebene genauso stellt wie auf der Bundesebene. Nimmt man die unterschiedlichen Zuständigkeiten in der Gesetzgebung als Gradmesser, muss die Antwort wohl nein lauten. Die Materien der Landespolitik, die wegen der Aufgabenverteilung im deutschen Föderalismus sehr verwaltungslastig sind, gestatten einen konsensuellen Stil eher als die ideologisch stärker aufgeladene Bundespolitik. Dem steht jedoch die parteipolitische Gleichförmigkeit der Landes- und Bundespolitik gegenüber. Diese ist ebenfalls eine Konsequenz des föderalen Systems, das die eigenverantwortliche Wahrnehmung der Länderkompetenzen zugunsten der Mitregierung auf Bundesebene zunehmend verschoben und damit die Eigenstaatlichkeit der Länder ausgehöhlt hat. Der Unitarismus spiegelt sich dabei zugleich in der Homogenität der Regierungsformen wider. Anders als die Kommunen, die in den achtziger und neunziger Jahren flächendeckend zum bis dahin nur in Süddeutschland geläufigen präsidentiellen Modell übergegangen sind, ahmen die Länder das parlamentarische Regierungssystem des Grundgesetzes bis ins Detail nach. ${ }^{42}$ Wenn aber die Länder vom Aufgabenzuschnitt her den Kommunen eher ähneln als dem Bund, warum könnten sie sich dann nicht auch bei der Gestaltung der Regierungssysteme an diesen orientieren? Der Wechsel zum präsidentiellen Modell hätte unter Demokratiegesichtspunkten gleich einen doppelten Vorteil: Einerseits würde man so die bundespolitische Überlagerung der Landtagswahlen zurückdrehen und der Landespolitik wieder ein stärkeres eigenständiges Gewicht einräumen, andererseits wäre - gleichsam als Nebeneffekt - eine bessere Integration der Volksgesetzgebung möglich. Das Pferd würde gewissermaßen von hinten aufgezäumt. Statt die Volksgesetzgebung dem bestehenden parlamentarischen System einzupassen, was in der Tendenz auf eine restriktive Ausgestaltung der Plebiszite hinausläuft, würde die Regierungsform auf das bestehende System der Direktdemokratie zugeschnitten. Die Volksrechte bekämen auf diese Weise einen breiteren Spielraum, könnten sich innerhalb des repräsentativen Systems als alternativer Pfad der Gesetzgebung leichter zur Geltung bringen.

Dass der Präsidentialismus eine besser geeignete Regierungsform für die Länder bereit halten könnte als das bestehende parlamentarische System, ist als Überlegung aus der Staatsrechtslehre und Politikwissenschaft nicht neu. ${ }^{43}$ Theodor Eschenburg ventilierte das

42 Vgl. Frank Decker, Die Regierungssysteme in den Ländern, in: ders. (Hrsg.), Föderalismus an der Wegscheide?, Wiesbaden 2004, S. 169 - 201.

43 Vgl. ebenda, S. 194 ff. Diese Erkenntnis wird allerdings nicht von allen geteilt. Die Kritiker weisen darauf hin, dass vor dem Hintergrund der fehlenden Gesetzgebungskompetenzen die Einführung des präsidentiellen Modells in den Ländern zu einer völligen Entmachtung des Parlaments führen würde, dem dann auch das Recht entzogen wäre, die Regierung zu bestellen und abzuberufen. Die von den Befürwortern unterstellte Nähe zur Kommunalpolitik wird mit Verweis auf die Beteiligung der Länder an der Bundesgesetzgebung ebenfalls bestritten. Dies habe zur Folge, dass die Länderpolitik sich den Zwängen des gesamtstaatlichen Parteienwettbewerbs gerade nicht entziehen könne. Ein präsidentielles System würde deshalb nur funktionieren, wenn der Ministerpräsident eine dauerhaft verlässliche parlamentarische Mehrheit hinter sich weiß. Genau dies sei aber durch die Entkoppelung von Parlaments- und Regierungswahl nicht gewährleistet. Für eine systematische Ausarbeitung des Direktwahlkonzepts, das auch die Gegenposition berücksichtigt vgl. Jan L. Backmann, Direktwahl der Ministerpräsidenten. Als Kern einer Reform der Landesverfassungen, Berlin 2006. 
Modell bereits zu Beginn der fünfziger Jahre, als es darum ging, eine Verfassung für den neu zu gründenden Südweststaat Baden-Württemberg zu entwerfen. ${ }^{44}$ Nachdem das parlamentarische System zu dieser Zeit in allen Ländern schon fest etabliert war, konnten sich seine Überlegungen damals nicht durchsetzen. Erst in den neunziger Jahren wurden sie von Hans Herbert von Arnim erneut aufgegriffen und öffentlich lanciert. ${ }^{45}$ Die unter Federführung des streitbaren Parteienkritikers zustande gekommene „Frankfurter Intervention“ fand jedoch wenig Widerhall - trotz des gleichzeitigen Siegeszugs der präsidentiellen Regierungsform in den Kommunen. Zudem erfolgte sie zu einem Zeitpunkt, als man die Weichenstellungen zugunsten des parlamentarischen Systems in den neuen Bundesländern längst vorgenommen hatte, wo die Gelegenheit für eine Reform noch am ehesten bestanden hätte. ${ }^{46}$ Auch eine Verfassungsinitiative der Freien Wähler für die Einführung der Direktwahl im Land Rheinland-Pfalz, die von Arnim mit betrieben hatte, versandete.

Mustert man die älteren Vorschläge für die Einführung des präsidentiellen Systems in den Ländern, so spielt die Integrationsproblematik der - seinerzeit wenig populären - Direktdemokratie darin noch keine Rolle. Auch von Arnim spricht den Zusammenhang nur indirekt an; für ihn stellen Direktwahl und Volksgesetzgebung gleichgelagerte Möglichkeiten dar, die „Allmacht“ der Parteien im politischen System zu brechen. ${ }^{47}$ Der Volksgesetzgebung kommt darüber hinaus die besondere Bedeutung zu, dass sie die Reformen selbst befördern kann - wenn nötig gegen den Willen der Regierenden. In der Vergangenheit haben sich die Reformanstrengungen weitgehend auf zwei Bereiche konzentriert: das Wahlrecht und die direkte Demokratie. Wo diese Anstrengungen erfolgreich waren - wie in Hamburg und Bremen - könnte als nächstes auch das parlamentarische System in das Visier des Volksgesetzgebers geraten. ${ }^{48}$ Dies gilt umso mehr, als die Stadtstaaten aufgrund ihres Doppelcharakters als Land und Gemeinde für eine solche Reform ohnehin prädestiniert wären.

\section{Beseitigung der Hindernisse durch Systemwechsel?}

Dem durchaus eindrucksvollen Ausbau der plebiszitären Instrumente in den Ländern auf der Ebene der förmlichen Verfassung steht ihre relative Bedeutungslosigkeit im realen Verfassungsleben weiterhin entgegen. Der Widerspruch rührt nicht nur aus der generellen Politikmüdigkeit der Bevölkerung, die sich gleichermaßen in Wahl- und Abstimmungsabstinenz äußert; er liegt auch in der verfassungsmäßigen Grundausrichtung der Direktdemokratie begründet, deren Ausgestaltung als Volksgesetzgebung weitgehende Restriktionen in

44 Vgl. Theodor Eschenburg, Verfassung und Verwaltungsaufbau des Südweststaates, Stuttgart 1952.

45 Vgl. Hans Herbert von Arnim, Wege aus der Krise des Parteienstaates. Thesen der „Frankfurter Intervention", in: Recht und Politik, 31. Jg. (1995), H. 1, S. 16-26.

46 Vgl. Carsten Giersch, Föderalismus und Länderparlamentarismus nach der deutschen Einheit, in: Peter R. Weilemann / Günter Buchstab / Hanns-Jürgen Küsters (Hrsg.), Macht und Zeitkritik. Festschrift für Hans-Peter Schwarz zum 65. Geburtstag, Paderborn / München / Wien / Zürich 1995, S. $637-648$.

$47 \mathrm{Vgl}$. Hans Herbert von Arnim, a.a.O. (Fn. 4), S. 258 ff.

48 Zum Zusammenspiel von Direktdemokratie und parlamentarischer Regierungsform am Hamburger Beispiel vgl. Frank Decker, Direkte Demokratie auf hanseatisch, in: MUT 2004, Nr. 442, S. $14-17$. 
der Verfahrenspraxis präjudiziert, die das Instrument de facto entwerten. Die Legitimation der Parteiendemokratie wird dadurch eher geschwächt als gestärkt.

Aus Sicht der pro-plebiszitären Kräfte ist es konsequent, durch eine Beseitigung der Restriktionen die Anwendbarkeit der Direktdemokratie so weit wie möglich zu verbessern. Weil sie dabei auf die direktdemokratischen Instrumente selbst zurückgreifen können, lässt sich dies nötigenfalls auch gegen den Willen der Regierenden durchsetzen. Konflikte zwischen dem parlamentarischen und dem Volksgesetzgeber werden damit jedoch unvermeidlich. Sie dürften gerade in den mehrheitsdemokratisch geprägten Systemen vorprogrammiert sein, die ihre parlamentarische Funktionslogik auf den Gegensatz von regierender Mehrheit und Opposition abstellen - ein Modell, das in der Bundesrepublik föderalismusbedingt auch auf der Ebene der Länder vorherrscht. Die Folgen sind Dauerstreitigkeiten um die rechtliche Zulässigkeit oder Unzulässigkeit der Verfahren und ein politisches Hin und Her zwischen anwenderfreundlicher und restriktiver Ausgestaltung, die die Akzeptanz der Direktdemokratie gefährden.

Theoretisch gäbe es zwei Möglichkeiten, um aus dieser Sackgasse herauszukommen. Die erste bestünde in einer Abkehr vom Volksgesetzgebungsmodell. Nach den Regeln der Pfadabhängigkeit kann dies mit ziemlicher Gewissheit ausgeschlossen werden. Nicht nur, dass die Abschaffung der bestehenden Volksrechte als demokratischer Rückschritt empfunden würde, der gegen die Mehrheitsmeinung der Bevölkerung kaum durchsetzbar wäre. Auch die Restriktionen des Modells weisen entwicklungsgesetzlich eher in Richtung einer (das Modell bekräftigenden) Verfahrenserleichterung, was durch den Verfassungsprozess in den deutschen Ländern bestätigt wird. ${ }^{49}$

Die andere Möglichkeit würde auf eine konsensuelle Transformation des Regierungssystems hinauslaufen. Dies könnte im Prinzip auf drei Wegen geschehen: erstens durch die Wirkungen der direkten Demokratie selbst (so wie in der Schweiz), zweitens durch einen Wandel des Parteiensystems und drittens durch eine andere Regierungsform, also den Wechsel vom parlamentarischen zum präsidentiellen Modell. Die Erfolgsaussichten der beiden zuerst genannten Wege scheinen begrenzt. Größere konsensuelle Wirkungen der Direktdemokratie sind nicht zu erwarten, solange diese im Regierungsgeschehen ein Randdasein fristet und auf die Ebene der Länder beschränkt bleibt. Auch die Pluralisierung des Parteiensystems dürfte nur allmählich zu einer Überwindung der gegnerschaftlichen Logik des Parteienwettbewerbs beitragen, die von den politischen Akteuren in der Bundesrepublik über Jahrzehnte hinweg internalisiert wurde. Symptomatisch dafür sind das Unvermögen, neue, lagerübergreifende Koalitionen zu bilden und die mangelnde Akzeptanz von Minderheitsregierungen. Die Forderung nach einem radikalen Schnitt, der die Sphären der

49 Eine andere Frage ist, ob nach den Regeln der Pfadabhängigkeit eine Übernahme des Volksgesetzgebungsmodells auch im nationalen Rahmen als ausgemacht gelten kann (wenn man den Entschluss fasst, Plebiszite dort einzuführen). Während die deutsche Verfassungstradition und der Ist-Zustand in den Ländern einen solchen Schluss nahelegen, sprechen die unterschiedlichen institutionellen Kontexte der Landes- und Bundespolitik dagegen. Auf der Bundesebene müsste zum Beispiel das Problem der Beteiligung der Zweiten Kammer an der Volksgesetzgebung zusätzlich gelöst werden, das in den Ländern entfällt. Hier mag einer der Gründe dafür liegen, warum sämtliche Versuche, Plebiszite in das Grundgesetz einzuführen, bislang im Sande verlaufen sind. Vgl. Frank Decker, Das Volk als Gesetzgeber? Zur Debatte um die Einführung plebiszitärer Elemente auf Bundesebene, in: Uwe Backes / Alexander Gallus / Eckhard Jesse (Hrsg.), Jahrbuch Extremismus \& Demokratie 2009, 21. Jahrgang, Baden-Baden 2010, S. 72 - 97. 
Bundes- und Landespolitik institutionell stärker voneinander trennt, gewinnt vor diesem Hintergrund an Plausibilität. ${ }^{50}$ Wer einen solchen Wechsel der Regierungsform als unrealistisch abtut, übersieht die Möglichkeiten, die den Verfassungsreformern in den Ländern gerade durch die direkte Demokratie zugewachsen sind.

50 In der Literatur wird zu Recht auf die Parallelen verwiesen, die in der Funktionslogik zwischen dem Minderheitsparlamentarismus und der präsidentiellen Regierungsform bestehen. Vgl. Nicole Bolleyer, Minderheitsparlamentarismus - eine akteurszentrierte Erweiterung der Parlamentarismus-/Präsidentialismustypologie, in: ZPol, 11. Jg. (2001), H. 4, S. 1519 - 1546.

\title{
Die Bedeutung von Eintragungsfristen beim Volksbegehren im Rahmen der Volksgesetzgebungsverfahren in den deutschen Ländern
}

\author{
Frank Meerkamp
}

Die frühe Bundesrepublik war eine „prononcierte antiplebiszitäre Repräsentativdemokratie" . Doch nicht die Weimarer Erfahrungen waren für den Parlamentarischen Rat ausschlaggebend, auf direktdemokratische Verfahren im Grundgesetz zu verzichten, sondern politische Gründe, die mit dem einsetzenden Kalten Krieg und der Teilung Deutschlands zusammenhingen. In den „aufgeregten Zeiten“2 sollte das zunächst als „Provisorium“ gedachte Grundgesetz ${ }^{3}$ nur vorübergehend mit einer „plebiszitären Quarantäne“ versehen werden. ${ }^{4}$ Die Mitglieder des Parlamentarischen Rates behielten dem späteren Verfassungsgeber mit Art. 20 Abs. 2 GG ausdrücklich eine plebiszitäre Option vor. Eine Reihe von Bundesländern nahm die Volksgesetzgebung bereits von Anfang an in ihre Verfassungen auf. Diese war allerdings meist restriktiv ausgestaltet und zeichnete sich durch hohe Unterstützungsquoren und kurze Einzeichnungsfristen beim Volksbegehren, teilweise auch Beteiligungs- oder Zustimmungsquoren beim Volksentscheid, aus - Hürden, die nur äußerst beschwerlich überwunden werden können. Dahinter stand durchaus der Gedanke, dass das Volk nur im Ausnahmefall als Korrektiv bei bedeutenden gesamtgesellschaftlichen Fragen eingreifen können sollte. Grundsätzlich herrschte ein etatistisch-korporatistisches Demokratieverständnis vor, das sich aber in den folgenden Jahrzehnten wandeln sollte und mit ihm auch die Ausgestaltung der Volksgesetzgebung.

1 Klaus Stern, Grundbegriffe und Grundlagen des Staatsrechts - Strukturprinzipien der Verfassung, München 1977, S. 455, S. 745.

2 Rudolf Katz, Parlamentarischer Rat, 3. Sitzung 1948, Stenographischer Bericht, S. 43.

3 Vgl. Heinrich Pehle, Probleme einer plebiszitären Ergänzung des Grundgesetzes, in: Gegenwartskunde, 47. Jg. (1998), H. 3, S. 299 - 310, S. 302.

4 Vgl. Otmar Jung, Grundgesetz und Volksentscheid, Opladen 1994, S. 331. 